\title{
A Novel 4-Methylideneimidazole-5-one-Containing Tyrosine Aminomutase in Enediyne Antitumor Antibiotic C-1027 Biosynthesis
}

\author{
Steven D. Christenson, ${ }^{\dagger}$ Wen Liu, ${ }^{\ddagger}$ Michael D. Toney, ${ }^{*, \dagger}$ and Ben Shen ${ }^{*,+, \neq, \S}$ \\ Department of Chemistry, University of California-Davis, Davis, California 95616, and Division of Pharmaceutical \\ Sciences and Department of Chemistry, University of Wisconsin-Madison, Madison, Wisconsin 53705
}

Received February 11, 2003; E-mail: mdtoney@ucdavis.edu; bshen@pharmacy.wisc.edu

C-1027, a chromoprotein natural product produced by Streptomyces globisporus, belongs to the enediyne family of antitumor antibiotics. ${ }^{1,2}$ The C-1027 chromophore (1) is characterized by an enediyne core, a deoxy aminosugar, a benzoxazolinate, and an $(S)$ 3-chloro-4,5-dihydroxy- $\beta$-phenylalanine moiety (2). We recently cloned and characterized the C-1027 biosynthetic gene cluster from S. globisporus and proposed that $\mathbf{2}$ is derived from L-tyrosine (3), implicating the involvement of an aminomutase in converting the $\alpha$-amino acid precursor into the $\beta$-amino acid intermediate (Figure 1). ${ }^{3}$ We now report that this reaction is catalyzed by $\mathrm{SgcC} 4$, a novel tyrosine aminomutase that converts 3 into $(S)-\beta$-tyrosine (4) during the biosynthesis of $\mathbf{1}$. We also demonstrate that it employs the 4-methylideneimidazole-5-one (MIO) cofactor, of which dehydroalanine is a component.

Aminomutases characterized to date fall into four groups: radical-based enzymes that use either cobalamin or $S$-adenosylmethionine plus an iron-sulfur cluster, ${ }^{4}$ pyridoxamine phosphatedependent enzymes, ${ }^{5}$ and ATP-dependent enzymes. ${ }^{6}$ An aminomutase exhibiting characteristics different from the above groups has also been reported recently but was only in the form of cellfree extracts; ${ }^{7}$ the exact nature of its catalytic mechanism remains to be determined.

None of the genes identified within the C-1027 gene cluster encode proteins with significant homology to known aminomutases. Instead, we identified $\operatorname{sgcC}_{\mathrm{C}}$, whose deduced product shows high homology, including the signature Ala-Ser-Gly motif, to a family of ammonia lyases. These include the histidine ammonia lyase from Streptomyces griseus $^{8}$ (39\% identity and 56\% similarity) and the phenylalanine ammonia lyase from Streptomyces maritimus ${ }^{9}(38 \%$ identity and $56 \%$ similarity). We inactivated $s g c C 4$ by replacing it with a mutant copy in which $\mathrm{sgcC} 4$ was disrupted by aac(3)IV according to published procedures. ${ }^{3}$ The resultant $S$. globisporus SB1007 mutant lost its ability to produce 1, confirming that $\mathrm{sgcC} 4$ is essential for the biosynthesis of $\mathbf{1}$. On the basis of these results, we proposed that $\mathrm{SgcC} 4$ is a novel tyrosine aminomutase that acts via $\alpha, \beta$-elimination of $\mathbf{3}$ to yield enzyme-bound ammonia and $p$-hydroxycinnamate (5), with subsequent Michael addition between ammonia and $\mathbf{5}$ to afford $\mathbf{4}$. This represents an unprecedented mechanism for an aminomutase (Figure 2).

To validate this hypothesis, the $\operatorname{sgcC} 4$ gene was amplified from pBS1005 3 by PCR, and the resultant product was cloned as an NdeI-HindIII fragment into the same sites of pET28a and sequenced to confirm PCR fidelity, yielding the expression construct pBS1022. ${ }^{10}$ Introduction of pBS1022 into E. coli BL-21(DE-3) resulted in the overproduction of $\mathrm{SgcC}_{4}$ as a $\mathrm{His}_{6}$-tagged fusion protein, which was purified by affinity chromatography on Ni-NTA resin. ${ }^{10}$ The purified SgcC4 $(0.5 \mathrm{mg} / \mathrm{mL})$ was incubated with $3(0.5 \mathrm{mM})$ in

\footnotetext{
University of California-Davis.
Division of Pharmaceutical Sciences, University of Wisconsin-Madison

$\$$ Department of Chemistry, University of Wisconsin-Madison.
}

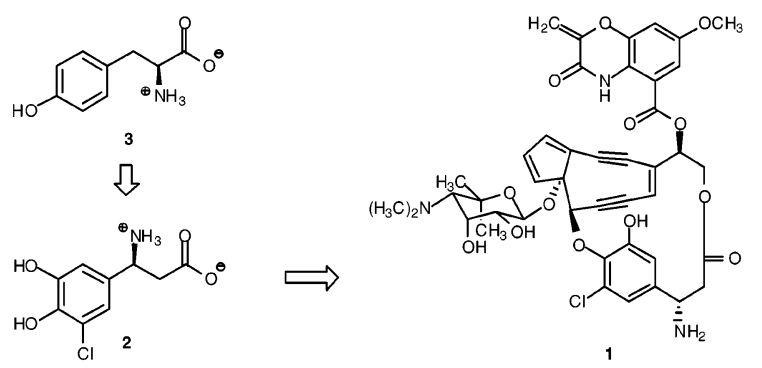

Figure 1. Structure of the C-1027 chromophore (1) and proposed biosynthetic origin of the $(S)$-3-chloro-4,5-dihydroxy- $\beta$-phenylalanine moiety (2) from L-tyrosine (3).
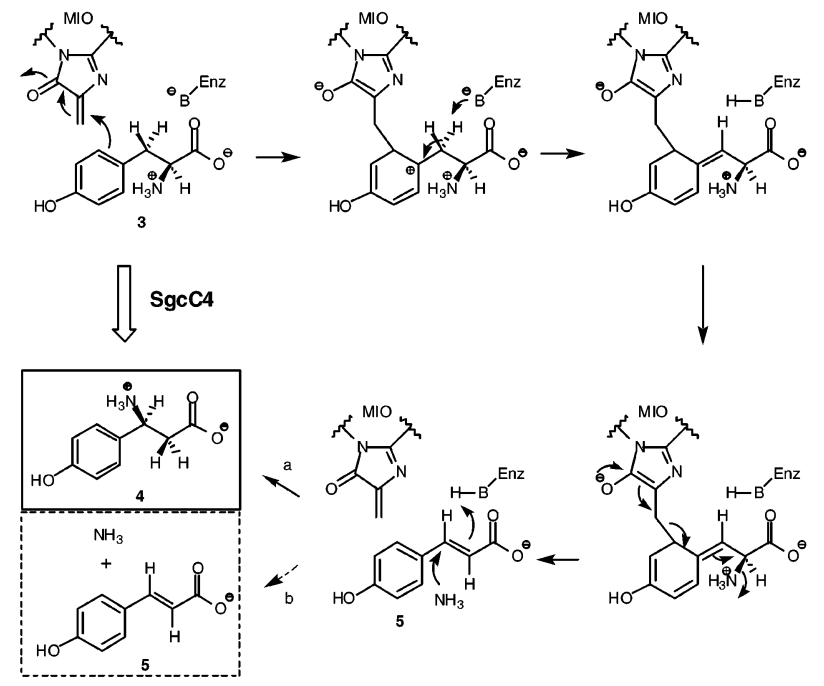

Figure 2. Proposed mechanism of the $\mathrm{SgcC} 4$ tyrosine aminomutase that catalyzes 3-to-4 conversion and employs MIO at its active site.

$100 \mathrm{mM}$ Tris- $\mathrm{HCl}, \mathrm{pH} 8.8$, at $25^{\circ} \mathrm{C}$ to test directly the aminomutase activity in vitro. The reaction was terminated by addition of $\mathrm{HCl}$ to $\mathrm{pH}<2$. The quenched mixture was derivatized with $o$ phthaldialdehyde ${ }^{11}$ and subjected to HPLC analysis. ${ }^{12}$ Baseline separation for $\mathbf{3}$ and $\mathbf{4}$ as $\boldsymbol{o}$-phthaldialdehyde derivatives and $\mathbf{5}$ was achieved (Figure 3, inset). As shown in Figure 3,>80\% of $\mathbf{3}$ was converted into $\mathbf{4}$ with $\mathbf{5}$ as a minor product $(<10 \%)$ after $180 \mathrm{~min}$ of incubation. The identities of $\mathbf{4}$ and $\mathbf{5}$ were verified by cochromatography with authentic standards and by characteristic UV absorption patterns. HPLC analysis using a chiral column ${ }^{13}$ with both authentic $(S)$ - and $(R)-\mathbf{4}$ as standards confirmed the enzymatic product to be $(S) \mathbf{- 4}$, as would be predicted from the stereochemistry of 1. These results unambiguously establish $\mathrm{SgcC} 4$ as a tyrosine aminomutase that catalyzes the conversion of $\mathbf{3}$ to $\mathbf{4}$. The fact that 5 was detected as a minor product provides direct evidence supporting the hypothesis that the $\mathrm{SgcC} 4$ catalyzed 3-to-4 conversion proceeds via $\mathbf{5}$ as an intermediate. 


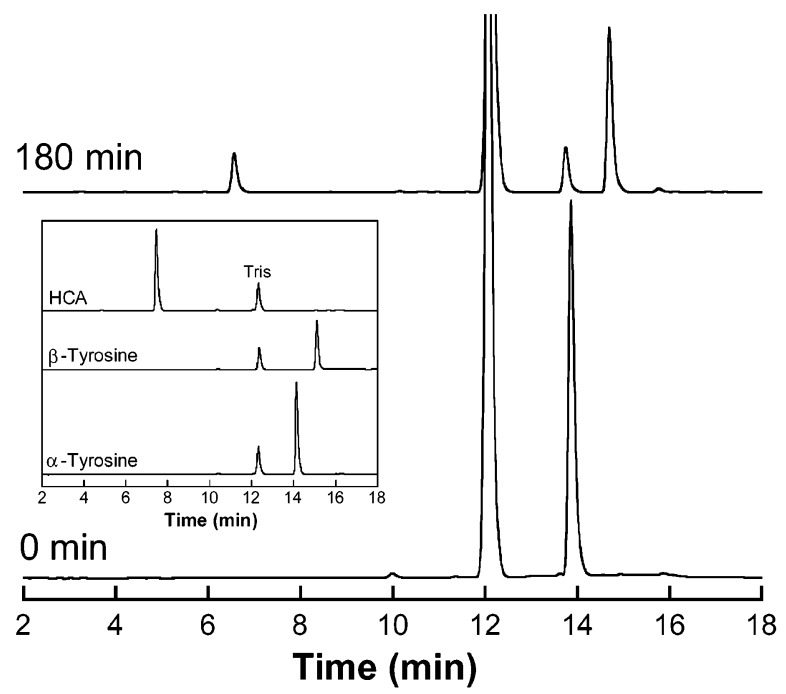

Figure 3. HPLC analysis of in vitro synthesis of $(S)-\beta$-tyrosine (4) from L-tyrosine (3) catalyzed by $\mathrm{SgcC} 4$. Traces for samples taken after 0 and $180 \mathrm{~min}$ of reaction are shown. Authentic standards are shown in the inset. The amino acids were analyzed as $o$-phthaldialdehyde derivatives and $p$-hydroxycinnamate (HCA) (5) in its underivatized form.

The active site of ammonia lyases was historically considered to contain a simple dehydroalanine residue until recent $\mathrm{X}$-ray crystallographic studies provided very strong support in favor of MIO that is formed autocatalytically from the conserved Ala-SerGly motif. ${ }^{14}$ The MIO group is known to be inactivated by borohydride and cyanide. ${ }^{15}$ To ascertain that the ScgC4 aminomutase utilizes MIO at its active site, we pretreated $\mathrm{SgcC} 4$ with either $10 \mathrm{mM} \mathrm{NaBH}_{4}$ or $2 \mathrm{mM} \mathrm{KCN}$. After removal of the excess reagents by filtration, the treated enzyme was assayed for tyrosine aminomutase activity. Consistent with the presence of MIO, pretreatment of $\mathrm{SgcC} 4$ with $\mathrm{NaBH}_{4}$ rendered the enzyme totally inactive, and $\mathrm{KCN}$ treatment of $\mathrm{SgcC} 4$ resulted in $>98 \%$ loss of its aminomutase activity. Inclusion of $\mathbf{3}(1 \mathrm{mM})$ during $\mathrm{KCN}$ pretreatment largely prevented the activity loss. In contrast, phenylalanine did not provide any protection against $\mathrm{KCN}$ inhibition, nor did it serve as a substrate for SgcC4. This implicates $\mathbf{3}$ as the precursor for $\mathbf{2}$ in the biosynthesis of $\mathbf{1}$.

Finally, to confirm that Ser153 from the conserved Ala-Ser-Gly motif of SgcC4 plays a critical role in the MIO-mediated 2,3aminomutase activity, we replaced Ser 153 with Ala by site-directed mutagenesis. ${ }^{16}$ The S153A mutation resulted in a 340-fold decrease in $k_{\text {cat }} / K_{\mathrm{M}}$ for $\mathrm{SgcC} 4$ as a tyrosine aminomutase. This compares well with the 430 -fold decrease in $k_{\text {cat }} / K_{\mathrm{M}}$ for phenylalanine ammonia lyase $\mathrm{e}^{17}$ and the 1170 -fold decrease in $k_{\text {cat }} / K_{\mathrm{M}}$ for histidine ammonia lyase ${ }^{18}$ engendered by the analogous mutation. The difference UV spectrum of SgcC4 and its S153A mutant exhibited a discrete absorption maximum around $308 \mathrm{~nm}$, characteristic for MIO. ${ }^{19}$ Taken together, these results strongly support the hypothesis that $\mathrm{SgcC} 4$ is an unprecedented aminomutase that requires $\mathrm{MIO}$ for activity.

Our finding that $\mathrm{SgcC} 4$ is a tyrosine aminomutase extends known MIO-dependent chemistry from simple ammonia lyases to that of aminomutases. On the basis of the MIO-dependent ammonia lyase paradigm, ${ }^{14}$ we envision that $\mathrm{SgcC} 4$ catalyzes the $\alpha, \beta$-elimination (i.e., ammonia lyase reaction) of $\mathbf{3}$ to yield ammonia and $\mathbf{5}$ as enzyme bound intermediates. Instead of releasing ammonia and $\mathbf{5}$ as the end products, as do ammonia lyases (Figure 2, path b), SgcC4 subsequently catalyzes a Michael addition of ammonia to the $\beta$-position of $\mathbf{5}$ (Figure 2, path a), acting overall as an aminomutase catalyzing the net 2,3-ammonia migration (Figure 2). The detection of $\mathbf{5}$ as a minor product apparently results from a competition between the Michael addition of ammonia to $\mathbf{5}$ and the release of the intermediates from the enzyme complex. It remains to be determined if this phenomenon is biologically relevant in vivo. Given the high sequence homology between $\mathrm{SgcC} 4$ and MIOdependent ammonia lyases, one wonders what the evolutionary relationship between $\mathrm{SgcC} 4$ and ammonia lyases is and whether the aminomutase activity could be engineered into ammonia lyases (and vice versa). One also wonders how widely MIO-dependent aminomutases are distributed in other organisms ${ }^{7}$ and what the metabolic advantages of having yet another type of aminomutase are. $^{4-6}$

Acknowledgment. We thank Dr. Y. Li, Institute of Medicinal Biotechnology, Chinese Academy of Medical Sciences, Beijing, China for the wild-type S. globisporus strain. This work was supported in part by NIH grant CA78747 (to B.S.). S.D.C. was supported in part by NIH grant T32 GM07377. M.D.T. acknowledges support from NIH (GM54779). B.S. is a recipient of an NSF CAREER Award (MCB9733938) and an NIH Independent Scientist Award (AI51689).

\section{References}

(1) (a) Otani, T; Minami, Y ; Marunaka, T; Zhang, R; Xie, M.-Y. Tetrahedron Lett. 1988, 41, 1580. (b) Yoshida, K.; Minami, Y.; Azuma R.; Saeki, M.; Otani, T. Tetrahedron Lett. 1993, 34, 2637.

(2) (a) Thorson, J. S.; Shen, B.; Whitwam, R. E.; Liu, W.; Li, Y.; Ahlert, J. Bioorg. Chem. 1999, 27, 172. (b) Brukner, I. Curr. Opin. Oncol., Endocr. Metab. Invest. Drugs 2000, 2, 344.

(3) (a) Liu, W.; Shen, B. Antimicrob. Agents Chemother. 2000, 44, 382. (b) Liu, W.; Christenson, S.; Standage, S.; Shen, B. Science 2002, 297, 1170.

(4) Frey, P. A.; Reed, G. H. Arch. Biochem. Biophys. 2000, 382, 6.

(5) Hennig, M.; Grimm, B.; Contestabile, R.; John, R. A.; Jansonius, J. N. Proc. Natl. Acad. Sci. U.S.A. 1997, 94, 4866.

(6) (a) Kurylo-Borowska, Z.; Abramsky, T. Biochim. Biophys. Acta 1972, 264, 1. (b) Parry, R. J.; Kurylo-Borowska, Z. J. Am. Chem. Soc. 1980, $102,836$.

(7) Walker, K. D.; Floss, H. G. J. Am. Chem. Soc. 1998, 120, 5333.

(8) Wu, P. C.; Korening, T. A.; White, P. J.; Kendrick, K. E. J. Bacteriol. 1992, 174, 1647

(9) Xiang, L.; Moore, B. S. J. Biol. Chem. 2002, 277, 32505.

(10) The construction of sgcC4 expression vector pBS1022 in pET28a, overproduction of SgcC4 by E. coli BL-21 (DE-3), and purification of $\mathrm{SgcC} 4$ by affinity chromatography were performed according to the manufacturer's protocols (Novagen, Madison, WI).

(11) Aberhart, D. J. Anal. Biochem. 1988, 169, 350.

(12) $o$-Phthaldialdehyde derivatives were loaded onto an Alltima C-18 column (5 $\mu \mathrm{m}, 150 \times 4.6 \mathrm{~mm}$, Alltech Associates, Deerfield, IL). The column was eluted with a 15 -min linear gradient from 10 to $65 \% \mathrm{CH}_{3} \mathrm{OH}$ (vol/ vol) in $50 \mathrm{mM} \mathrm{NaOAc}$, pH 5.7, with $5 \% \mathrm{THF}$ and held isocratically for an additional $5 \mathrm{~min}$, at a flow rate of $1.0 \mathrm{~mL} / \mathrm{min}$ with UV detection at $330 \mathrm{~nm}$. The column was washed with $\mathrm{CH}_{3} \mathrm{OH} / \mathrm{CH}_{3} \mathrm{CN}(1 / 1$, vol/vol) for $5 \mathrm{~min}$ between each sample.

(13) Filtered $\mathrm{SgcC} 4$ reaction samples were loaded onto a Chirobiotic-T column (5 $\mu \mathrm{m}, 250 \times 4.6 \mathrm{~mm}$, Astec Inc., Whippany, NJ) and eluted isocratically with $\mathrm{CH}_{3} \mathrm{OH} / \mathrm{AcOH} / \mathrm{Et}_{3} \mathrm{~N}(100 / 0.1 / 0.1$, vol/vol $)$, at a flow rate of $1.0 \mathrm{~mL} /$ min with UV detection at $280 \mathrm{~nm}$.

(14) Schwede, T. F.; Retey, J.; Schulz, G. E. Biochemistry 1999, 38, 5355.

(15) Silverman, R. B. The Organic Chemistry of Enzyme-Catalyzed Reactions; Academic Press: San Diego, 2000; pp 424-428.

(16) Site-directed mutagenesis using the Quickchange method was performed according to the manufacturer's protocol (Stratagene, La Jolla, CA).

(17) Langer, M.; Pauling, A.; Retey, J. Angew. Chem., Int. Ed. Engl. 1995, 34, 1464.

(18) Rother, D.; Poppe, L.; Morlock, G.; Viergutz, S.; Retey, J. Eur. J. Biochem. 2002, 269, 3065.

(19) Röther, D.; Merkel, D.; Rétey, J. Angew. Chem., Int. Ed. 2000, 39, 2462. JA034609M 